\title{
Sea Ice Image Processing Research Based On GOCI Satellite
}

\author{
ShengWen Gong ${ }^{1, a}$ \\ ${ }^{1}$ Qingdao University of Science \& Technology, SongLing Rd. 99, \\ 266071 Qingdao, China \\ agsw780604@126.com
}

\begin{abstract}
The GOCI (Geostationary Ocean Color Imager) satellite with high spatial resolution and high temporal resolution can satisfy the needs of monitoring drift of sea ice to provide real-time and high frequency data. The real-time and accurate monitoring information can be obtained to support the economic activities related with sea. The SIFT (Scale-invariant feature transform) algorithm is improved to realize two optimization schemes of the automatic selection of threshold and manual elimination of edge points to make use of the GOCI satellite image information effectively. The improved SIFT algorithm is utilized to extract the feature points of images of sea ice and match images. The feasibility and practicability of the improved algorithm is verified through comparison between the improved algorithm and conventional algorithm.
\end{abstract}

Keywords: Sea Ice Monitoring; Satellite Remote Sensing; SIFT Algorithm; Feature Extraction; Image Matching

\section{Introduction}

Sea ice monitoring of polar and high latitudes district is mainly based on microwave remote sensing, and the visible light and infrared are complementary manner. The remote sensing data used for sea ice monitoring and forecasting is mainly from NOAA/AVHRR、MODIS、ERS and RADARSAT. Scharfen et al use MODIS data to obtain the global ice and snow product picture [1]. Drue and Heinemann et al utilize MODIS data to get the information of the polar sea ice concentration [2]. The current monitoring means of sea ice mainly combine the space satellites, aircraft, icebreaker investigation and bank station, fixed platform and so on. The researchers in Beijing Normal University apply NOAA/AVHRR data to extract the range and the thickness of ice automatically to estimate the 
amount of sea ice of Liaodong Gulf [3]. There are some other manners to monitor or measure the movement state of sea ice such as radar monitoring, sonar surveying and video etc. But the common problem of theses manners is the need of installing the corresponding equipment, which is with a higher requirement in manpower and material resources. The measuring range of these manners is small to access the monitoring result of sea ice drift with a large area. So the manner to access the drifting direction and speed of sea ice by satellite remote sensing is practically significant to overcome the poor work environment of cold ocean field $[4,5]$.

GOCI satellite with high spatial resolution and high temporal resolution can satisfy the needs of monitoring sea ice drift to provide real-time and high frequency data for monitoring sea ice drift and provide real-time and accurate information of monitoring sea ice for economic activities in the ice area. Three optimization schemes to SIFT algorithm are proposed based on some existing feature points extraction and matching algorithm to research sea ice images of Huanghai Sea and Bohai Sea based on GOCI satellite. The availability and practicability of the improved algorithm is verified.

\section{Extraction algorithms of ice feature points}

There are two stages in the extraction algorithm of feature points. The first one is image segmentation. Extract the target area or its border and extract the basic characteristics such as gray scale, texture and shape description of the target. The second stage is to find or construct a target as feature invariant of image feature which is invariant when the target does some kind of exercise or no matter what kind of movement. When the number of extracted feature points is too much, there will be large amounts of interference information such as error matching points. The interference information will affect the accuracy and speed of matching. When the number of extracted feature points is too small, it will make bad effect on precise matching. The method of feature points matching needs a lot of efficient points in the two image point sets. The complexity of algorithm is increased when the number of image feature points is more.

SIFT extraction method of local invariant feature points. Extraction method of local invariant feature points mainly applies the information of local area to structure characteristic. As a result of these local areas may exist in different positions of the target discretely, the part information of the target can be gotten when extracting characteristics of each region independently even if the target is located in the complex environment or partial occlusions. So the 
identification and confirmation of target in complex background can be realized. Since the algorithm is without the pretreatment such as segmentation or extraction the target contour and does not need to get all the information of the target, it is more widely applicable than the global method. The feature points extracted by SIFT extraction method of local invariant feature points have better robustness to the image scale, rotation, light and changes of noise.

The steps of SIFT feature points extraction and testing. The mainly perform processes of SIFT extraction algorithm of feature points are shown in figure 1.

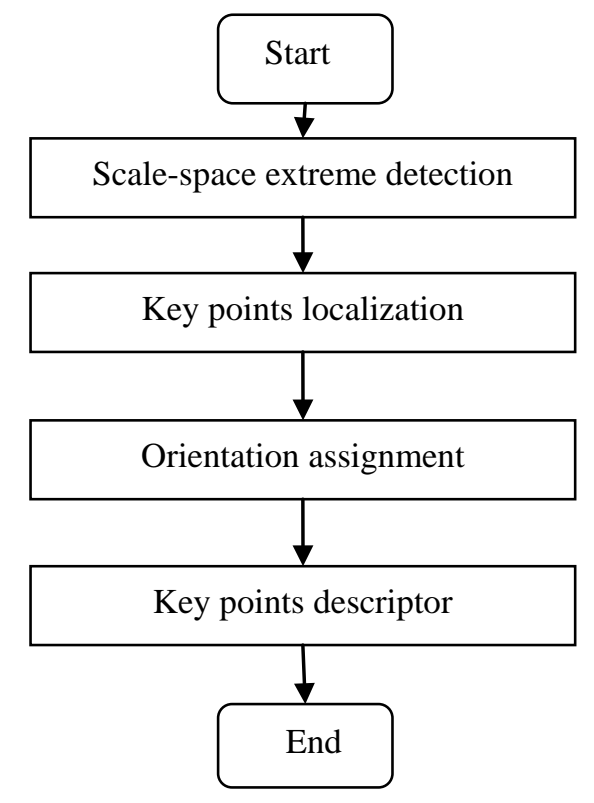

Fig. 1 Flow chart of SIFT algorithm

\section{Feature points matching of sea ice}

Matching method based on image characteristics can overcome the shortcomings existed in the matching method utilized the gray level information of image. Since the feature points of image is less than the total pixels of image, the amount of calculation in matching process is greatly reduced. The extraction process of feature points can reduce the influence of noise, the change of gray level, image deformation and block of image. 
The nearest neighbor matching algorithm based on Euclidean distance. The Euclidean distance of feature vector of key points is adopted to measure the similarity of key points of the two images after the SIFT feature vector of two images is generated. Take some critical point in the image 1 and find two points with nearest Euclidean distance with it in the image 2. In the two key points in image 2, if the value of the nearest distance divided by the second nearest distance is less than a threshold value, accept the pair of matching point. Reducing the threshold will make the number of matching point decrease, but make the matching more stable.

Lowe [6] proposed the method to compare the nearest neighbor distance with the second nearest neighbor distance to get rid of the key point without matching relation generated by image sheltering or confusion background. If the distance ratio is less than one threshold, the matching is considered right. In the error matching, the distance ratio is large because there may be a lot error matching for the high dimensional feature space.

Implementation steps of SIFT matching algorithm of feature points.

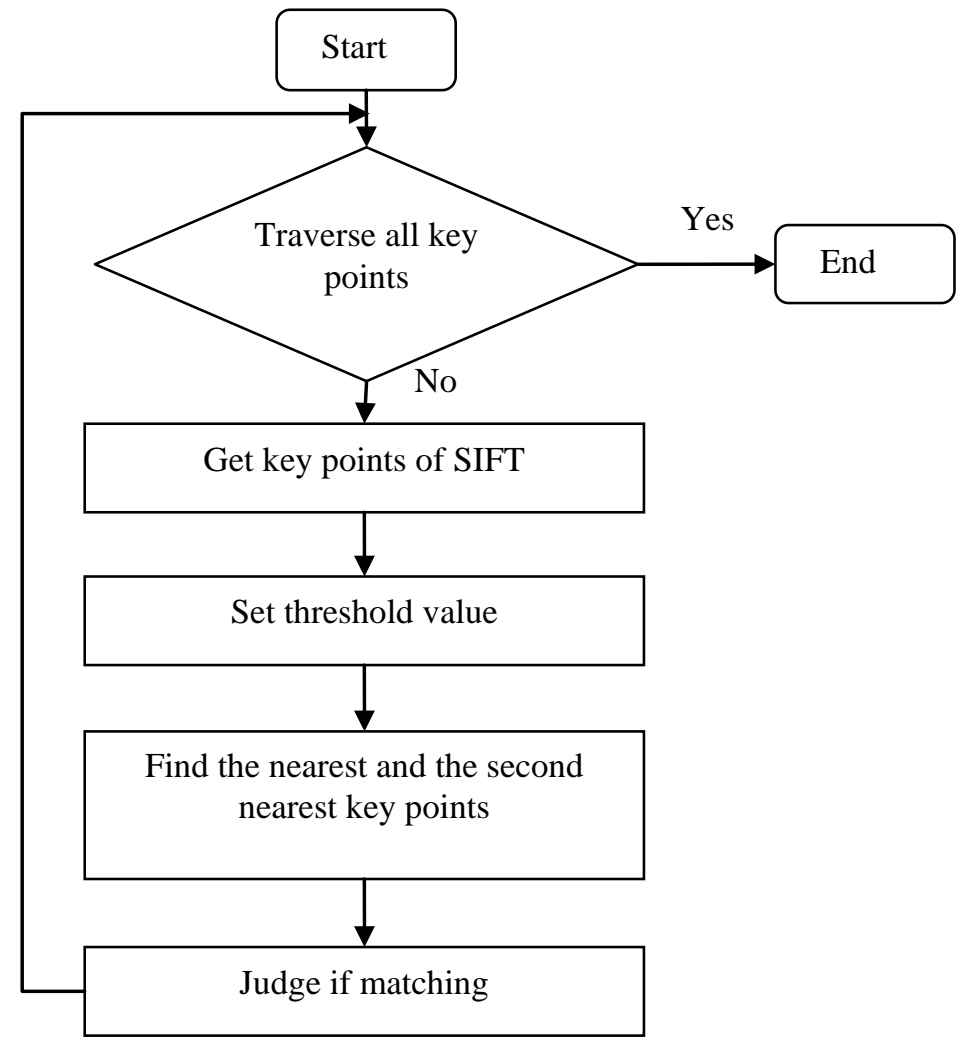


Fig.2 Flow chart of SIFT algorithm

\section{SIFT matching algorithm of feature points and its improvement}

Select threshold automatically. It can be concluded by the analysis of SIFT feature extraction algorithm of classical local invariant that the feature descriptor is a 128-dimensional vector. The corresponding feature points in the two pictures are matched through similarity measure of the feature descriptor of feature points. The setting of the distance ratio is the most direct factor which affects the matching result. Usually, the threshold value is set empirically, so it needs to calculate the extremum of distance ratio of all key points first, and then calculate a new threshold according to the extremum.

Remove edge points manually. Although some edge points and key points with low contrast have been deleted by SIFT algorithm, some other error matching occurs at the key edge points in some experiment test. Removing edge points manually refers to skip some matching process of the feature points at the location of edge of candidate point matrix when the matching algorithm executes. The program reduces the error matching of edge feature point but deletes some effective feature points.

\section{Example of handling feature points of the Huanghai Sea and the Bohai Sea}

The experiment platform is MATLAB 7.1. The image data source is the sea ice image of the Huanghai Sea and the Bohai Sea of GOCI satellite. The range of shooting is $117.9286{ }^{\circ} \mathrm{E} \sim 123.03334^{\circ} \mathrm{E}$ and $38.4921^{\circ} \mathrm{N} \sim 41.1096{ }^{\circ} \mathrm{N}$. The shooting time interval of two sea ice images is 1 hour. The two kinds of optimization scheme of SIFT matching algorithm of feature points are tested. The matching feature points in the image are connected by straight line.

Select threshold automatically. Test results are shown in table 1 . If the number of extracted image feature points is invariant, the number of matching pairs and error matching decrease with the decrease of the threshold gradually to be stable. There is obvious error matching in (a) of Figure 3, when the threshold is greater than or equal to 0.8 . The error matching is not obvious but the matching pairs are obviously decreased in (b), when the threshold is less than or equal to 0.4. The threshold of experiment image is 0.628901, when the threshold is chosen 
automatically. At this time, the number of matching pairs is more, and the number of error matching is moderate. So the result is ideal.

Table 1 Matching results when threshold value changes

\begin{tabular}{ccccc}
\hline $\begin{array}{c}\text { Threshol } \\
\mathrm{d}\end{array}$ & $\begin{array}{c}\text { Extracted } \\
\text { point of image } \\
1\end{array}$ & $\begin{array}{c}\text { Extracted } \\
\text { point of image } \\
2\end{array}$ & $\begin{array}{c}\text { The number of } \\
\text { matching pairs }\end{array}$ & $\begin{array}{c}\text { The number of } \\
\text { error matching } \\
\text { pairs }\end{array}$ \\
\hline 0.8 & 2138 & 2137 & 784 & 23 \\
0.7 & 2138 & 2137 & 636 & 7 \\
0.6 & 2138 & 2137 & 509 & 5 \\
0.5 & 2138 & 2137 & 351 & 3 \\
0.4 & 2138 & 2137 & 207 & 3 \\
0.3 & 2138 & 2137 & 89 & 3 \\
0.628901 & 2138 & 2137 & 546 & 5 \\
\hline
\end{tabular}
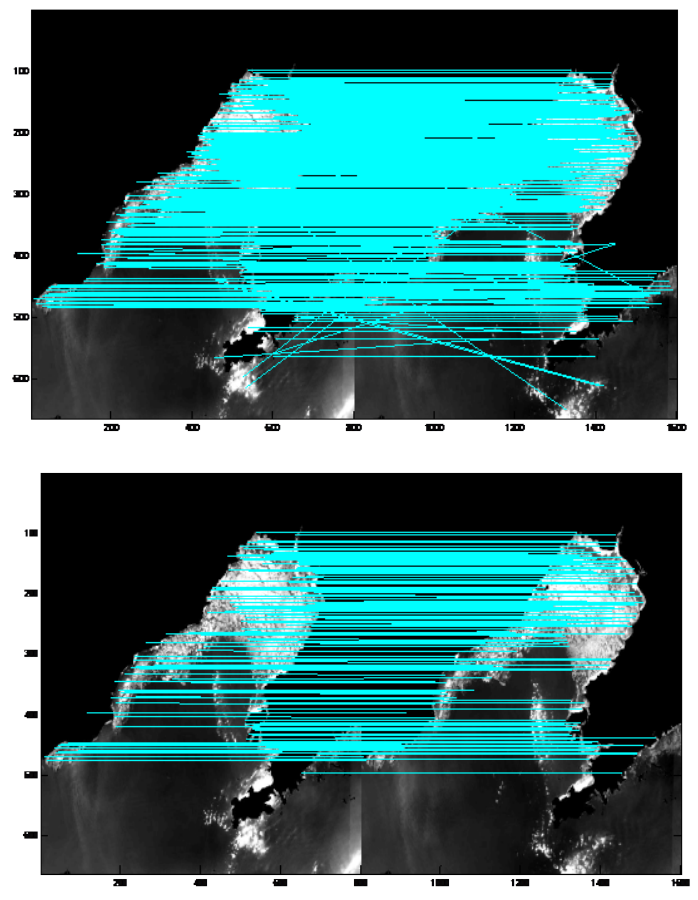

$\begin{array}{ll}\text { (a ) The matching result when threshold is } 0.8 & \text { (b) The matching }\end{array}$ result when threshold is 0.4 


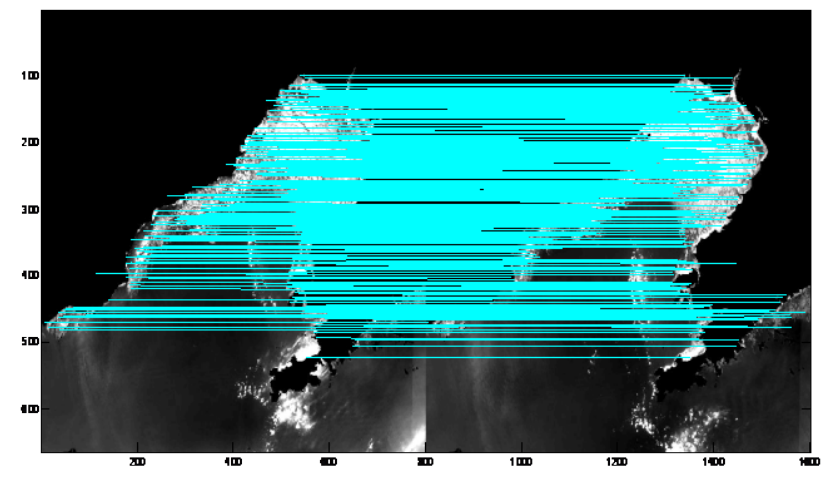

(c) The matching result when threshold is 0.628901

Fig.3 Matching result on different threshold values

Remove edge points manually. From above analysis on threshold (distance ratio), it can be concluded that there are always some error matching at edge of image which can not be eliminated through adjusting the thresholds, when the sea ice images are matched. Some feature points at the edge can not be matched to eliminate the edge points. 30 feature points of pixel location is not matched in the experiment. The specific test results are shown in Figure 4.

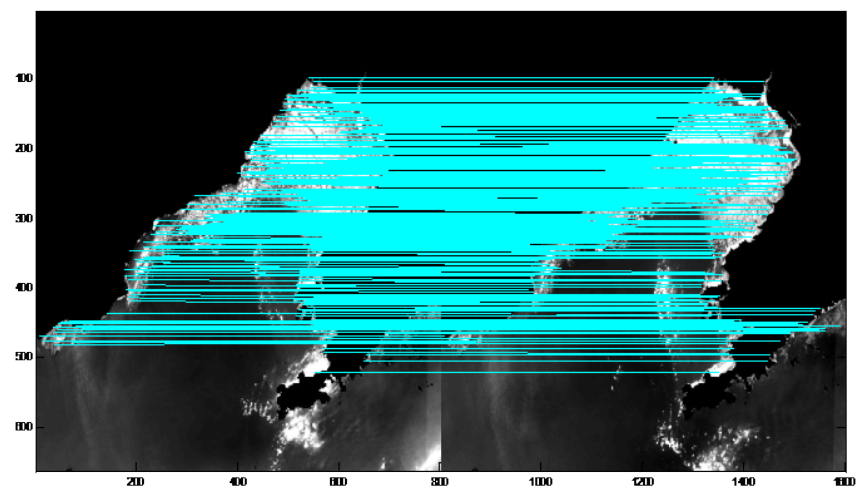

Fig.4 Matching result after eliminating the edge point

As shown in Figure 4, the number of matching pairs is decreased from 546 to 527, but the error matching at the edge is eliminated successfully. The elimination of error matching of feature points at the edge on the premise of almost not influence the matching rate is successful. 


\section{Conclusions}

The extraction and matching algorithms of image feature points are researched in this paper. The SIFT algorithm is improved to realize the two optimization schemes of ensuring threshold automatically and eliminating edge points manually. The improved SIFT algorithm is applied to extract feature points and match images on the sea ice images. The improved algorithm is more feasible and practical compared with the algorithm before.

\section{Acknowledgements}

This work was financially supported by the Open fund project of Shandong Provincial Key Laboratory of Marine Ecological Environment and Disaster Prevention and Mitigation (2012006), science and technology plan project of the colleges and universities in Shandong province (J14LN74).

\section{Reference}

[1] Scharfen G. and S. Khalsa. Assessing the Utility of MODIS for Monitoring Snow and Sea Ice Externt. Proceedings of Earsel-lissing-workshop Observing our Cryosphere from Space, 2002,11(13) :122 127.

[2] Drue, C., and G. Heinemann. High-resolution maps of the sea-ice concentration from MODIS satellite data. Geophys Res. Lett., 2004,31:L20403 1-5.

[3]Xie Feng, Gu Wei, Yuan Yi and Chen Yunhao. ESTIMATION OF SEA ICE RESOURCES IN LIAODONG GULF USING REMOTE SENSING [J]. RESOURCES SCIENCE, 2003,25(3): 17 23.

[4]Dorko G.,Schmid C. Selection of Scale Invariant Neighborhoods for Object Class Recognition[C]. In: Proceedings International Conference on Computer Vision,2003.634 640.

[5] David G Lowe. Object recognition from local scale-Invariant features[C]. In: Proceeding of the International Conference on Computer Vision (ICCV’1999), 1999.20 27.

[6] David G Lowe. Distinctive image features from scale-invariant keypoints[J]. International Journal of Computer Vision,2004,60(2): 91 110. 